\title{
Neurobehcet pediátrico. Presentación de un caso y revisión de la literatura.
}

\author{
Pediatric Neurobehcet. A case report and review of the literature.
}

\author{
Visaga Maribel*, Campos Patricia**, Bancalari Ernesto**, Romero Félix, \\ Hernandez Herminio***, Berrocal Alfredo, Trelles Luis**, Calvo Armando*. \\ *Unidad de Inmunología y Reumatología. Hospital Nacional Cayetano Heredia. \\ **Servicio de Neurología. Hospital Nacional Cayetano Heredia. \\ ***Departamento de Pediatría. Hospital Nacional Cayetano Heredia.
}

\section{SUMMARY}

The first peruvian pediatric case of Neuro-Behcet, who had seizures, hemiparesis and a magneic nuclear resonance with a pseudotumoral imagine is here presented (Rev Med Hered 1996; 7: 178-181).

KEY WORDS: Behcet, magnetic nuclear resonance, hemiparesis, seizures.

\section{INTRODUCCION}

En 1937 Hulusin Behcet, dermatólogo turco, describió una enfermedad en la cual úlceras orales y genitales se asociaban a uveitis. Han transcurrido más de 50 años desde aquella descripción y actualmente se reconoce que la enfermedad es una entidad crónica multisistémica, con un proceso patológico subyacente-vasculitis (1).

La enfermedad afecta mayormente adultos jóvenes entre 25 y 35 años siendo infrecuente en la niñez y en pacientes mayores de 50 años $(1,2)$. Por ejemplo, en la literatura peruana no hemos encontrado ningún caso pediátrico.

Entre sus diversas manifestaciones, el compromiso neurológico adquiere singular importancia, pués se asocia con un pronóstico reservado $(3,4)$. 
Reportamos una paciente de 16 años quien desarrolló compromiso severo del sistema nervioso central (SNC) y se revisa la literatura concerniente al tema.

\section{Caso Clínico}

Paciente mujer, de 16 años de edad, natural y procedente de Lima, estudiante, de raza mestiza; en abril de 1995 acude al Hospital Nacional Cayetano Heredia (HNCH) por presentar un cuadro de 45 días de evolución caracterizado por úlceras orales y genitales caracterizado por úlceras orales y genitales dolorosas, fiebre, artritis de codos y rodilla; y 7 días antes de ser admitida, disminución de agudeza visual. Al llegar a la consulta presentó convulsiones tónico-clónicas generalizadas acompañadas de relajación de esfinteres, con posterior inestabiliadad de la marcha y desarrollo en las 24 horas siguientes de hemiparesia derecha a predominio fasciobranquial. Refería la madre que 2 meses antes la paciente había abandonado la medicación que venía recibiendo por anterior diagnóstico de Behcet.

Antedecentes: Hace cuatro años la paciente presentó cuadro de alza térmica, convulsiones, poliartritis de grandes y pequeñas articulaciones, y úlcera dolorosa en la cavidad oral, siendo sometida a estudio en el Hospital Arzobispo Loayza de Lima y recibiendo tratamiento con penicilina EV, siendo dada de alta mejorada. Persistió con poliartritis por 2 años aproximadamente, después de los cuales presentó ataxia cerebelosa y cuadro convulsivo tocicolónico, úlceras genitales y orales dolorosas, por la cual es admitida al $\mathrm{HNCH}$, donde se hace el diagnóstico de Enfermedad de Behcet y se instaura tratamiento con prednisona $40 \mathrm{mg} /$ día siendo dada de alta con mejoría notable; y luego controlada ambulatoriamente con descenso de la prednisona.

Posteriormente presentó trastorno del pensamiento con ideas obsesivas e indiferencia al medio, además de uveitis anterior con presencia de hipopion; lo que motivó un incremento de la dosis de prednisona. La evolución fue ondulante, con período de exacerbación asociados a intento de disminución de dosis de corticoides; por ello en febrero de 1993 se añadió metotrexate (MTX) a $7.5 \mathrm{mg} / \mathrm{semana}$ vía oral, el que recibió por un año sin lograr disminución de la dosis de corticoides y control total de la enfermedad. Se suspendió MTX y se inició clorambucil a $6 \mathrm{mg} /$ día, lográndose disminuir la dosis de prednisona a mg/día sin reaparición de síntomas.

Al examen físico se encontró PA: 120/80 mm Hg, FC: 100/min y T:39 C. La paciente se encotraba en MEG, BEN, orientada en persona, aspecto cushingoide y lucía agudamente enferma. Se encontró máculas eritematosas en cara anterior de muslo izquierdo, sinovitis en rodillas y úlceras dolorosas en labios. El examen cardiopulmonar fue normal y no se encontró úlceras en los genitales.

El examen neurológico mostró hemiparesia derecha severa flácida con hiperreflexia y hemihipoestesia derecha, Babinski $(+)$ derecho, cuadrantopsia superior derecha a la campimetría por confrontación y ptosis palpebral derecha.

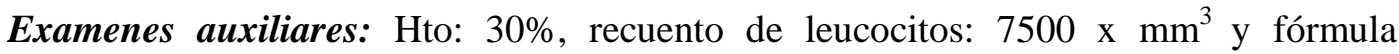
leucocitaria normal. VSG en $56 \mathrm{~mm}$ a la hora, hemocultivo, urocultivo y BK negativos, aglutinaciones tíficas, paratíficas y brucelares negativas, examen de orina normal y electrolitos séricos normales. La tomografía axial computarizada (TAC) cerebral mostró una lesión hipodensa de bordes irregulares de 3.6 x 3.7 cm de diámetro en región 
talámica y ganglios basales de hemisferio cerebral izquierdo que se extendía hasta la región temporo-occipital y desplazaba discretamente el III ventrículo hacia la derecha. La resonancia magnética nuclear (RNM) cerebral mostró un área difusa de hiposeñal en T1 e hiperseñal en T2 desde la unión bulbomedular, todo el trayecto de trococerebral hasta el pedúnculo cerebral cápsula interna, cápsula externa y de claustrum. La hiposeñal difusa producía leve efecto de masa distorsionando el pedúnculo cerebral. Se observó captación de contraste nodular a nivel subtalámico con mínima captación en superficie del tronco y pedúnculos cerebrales (Foto $\mathrm{N}^{\circ} 1$ ).

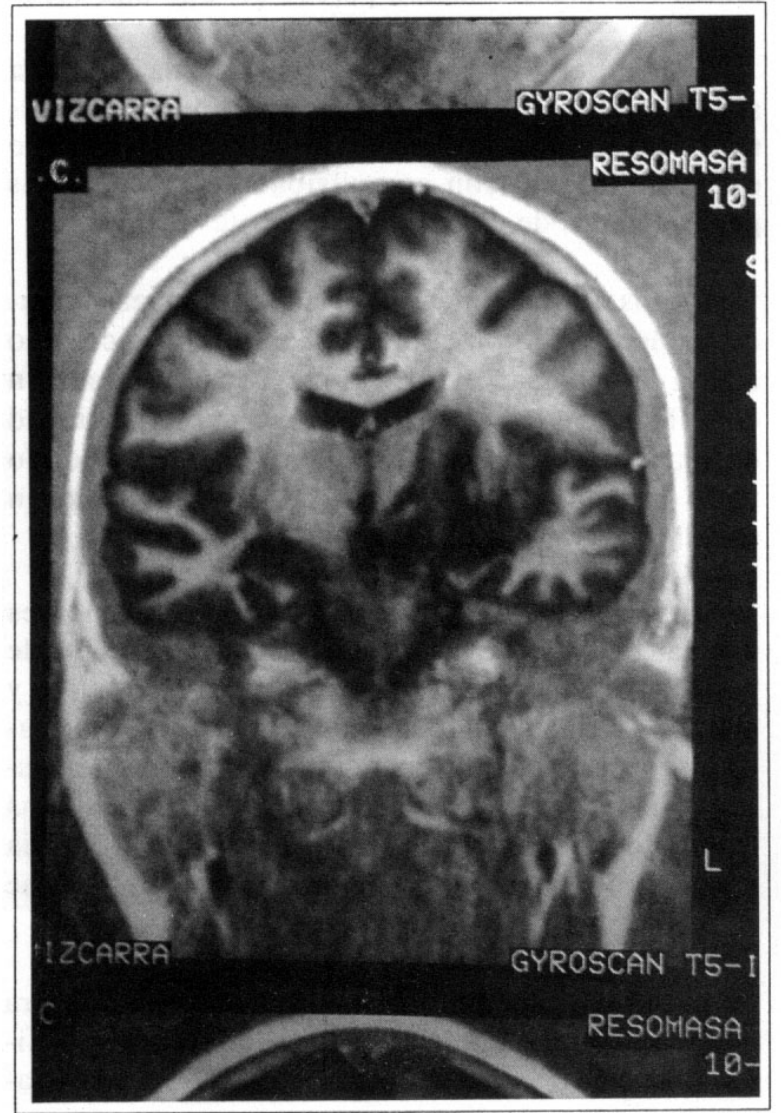

Tratamiento y evolución: La paciente recibió prednisona a $1 \mathrm{mg} / \mathrm{kg} / \mathrm{d}$, pulso de metilprednisolona, clorambucil a $10 \mathrm{mg} / \mathrm{kg} / \mathrm{d}$, pulso de metilprednisolona, clorambucil a $10 \mathrm{mg} /$ día e INH $300 \mathrm{mg} / \mathrm{d}$. Se observó evolución favorable, con desaparición de la ataxia, la hemiparesia derecha y la convulsiones. Los controles posteriores mostraron mejoría notable, decidiéndose disminuir la dosis del clorambucil y prednisona. Una RMN de control seis meses después, reveló lesiones cicatrizales en el tronco cerebral, diencéfalo y putamen izquierdos, demostrando mejoría respecto a RMN previa (Foto $\mathrm{N}^{\circ} 2$ ). 


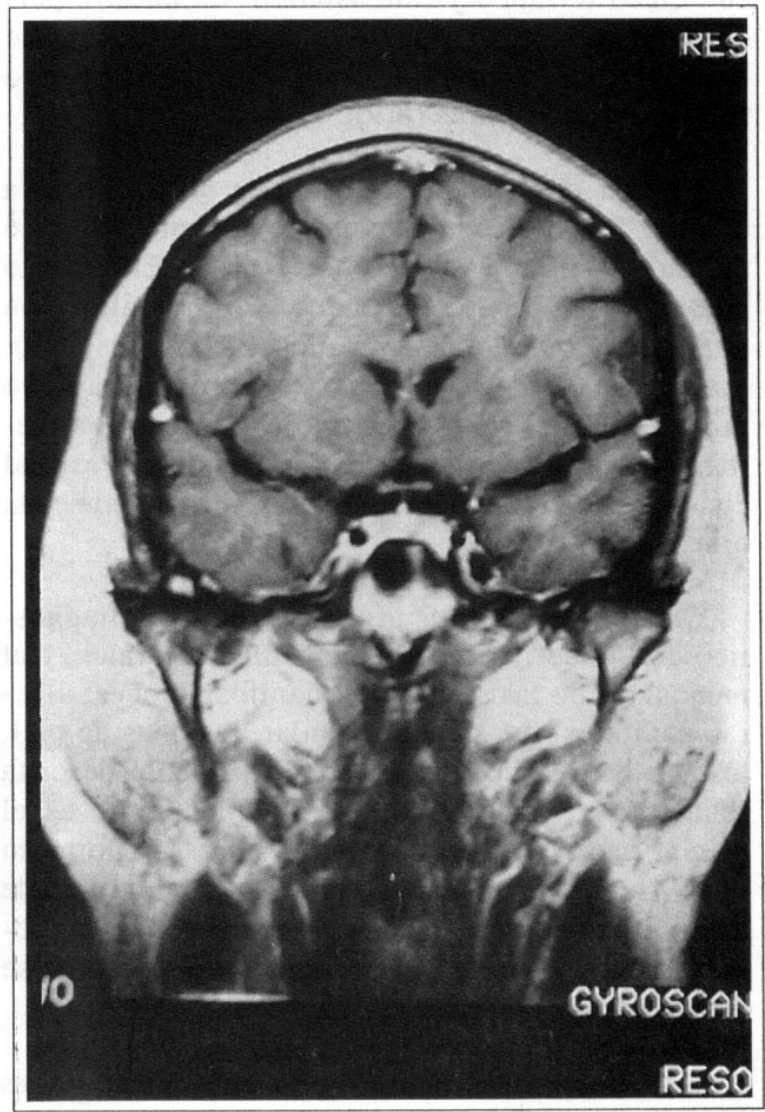

\section{DISCUSION}

La enfermedad de Behcet es multisistémica y de distribución mundial, aunque con mayor prevalencia en el mediterráneo y este asiático. En nuestro país existen escasos trabajos sobre esta entidad, los que describen sólo reportes de casos $(5,6,7)$ o un número pequeño de pacientes $(8,9)$.

Es una enfermedad que afecta mayormente a adultos jóvenes, con mayor prevalencia del sexo masculino en una relación 2:1 (1,2). En nuestro medio los 10 casos reportados fueron adultos con una edad promedio de 35.7 años (20-57 años) (5,9), y a diferencia de lo descrito en la literatura hubo predominio del sexo femenino (6/10 fueron mujeres). Ninguno de estos casos fue pediátrico, lo cual concuerda con lo señalado, pués es infrecuente en la niñez $(1,3)$.

Siendo los criterios propuestos para el diagnóstico de Behcet, criterio mayor: úlceras orales, y al menos dos de las siguientes manifestaciones: úlceras genitales, inflamación ocular, lesiones de la piel, test de pathergia; vemos que la paciente descrita reúne los requisitos para el diagnóstico y es el primer caso en edad pediátrica que se reporta en nuestro medio, además de tener la peculiaridad de un severo compromiso neurológico ocasionado probablemente por vasculitis, el que generalmente se asocia a un pronóstico reservado $(3,4,11)$. 
El compromiso neurológico en Behcet no es infrecuente, describiéndose en adultos con una frecuencia que varía entre $5-49 \%(2,4,12)$; en niños también se ha reportado casos de compromiso neurológico señalándose frecuencia tan altas como 60\% $(13,14)$.

Las manifestaciones más comunes son meningoencefalitis y lesiones a nivel del tronco encefálico; también se describen hemiplejía, disturbios visuales (usualmente causados por uveitis o vasculitis retiniana y raramente por neuritis óptica), hipertensión intracraneana como resultado de trombosis de los senos venosos cerebrales, compromiso cerebeloso y neuropatía periférica $(2,4,8,11,12)$. Nuestra paciente desarrolló convulsiones, alteraciones visuales por uveitis y ataxia cerebelosa al inicio de su enfermedad y años después hemiplejia y lesión de masa a nivel del tronco encefálico y ganglios basales que remitieron con el tratamiento.

Se ha señalado que las convulsiones focales o generalizadas pueden presentarse en el 5\% de los casos con compromiso neurológicos (15). Sin embargo Serdaroglu et al (12), en un estudio prospectivo no reportaron convulsiones en 17 pacientes con afección neurológica. Los hallazgos de Bertolet et al (16) concuerdan con estos datos. Por lo tanto, parece que este síntoma se presenta raramente. En niños, esta manifestación parece ser aún más rara.

Samoud et al, reportan el caso de una niña con status epiléptico y fiebre recurrente como manifestaciones de enfermedad de Behcet (17). Nuestro caso se presentó con convulsiones tonicoclónicas generalizadas de carácter igualmente recurrente.

De los 10 pacientes reportados en nuestro medio, sólo 2 tuvieron compromiso neurológico, uno ataxia cerebelosa y el otro polineuropatía y afección del VII par; nuestra paciente tuvo mayor variedad de manifestaciones neurológicas, teniendo por lo tanto un pronóstico más reservado y requiriendo un tratamiento más agresivo.

De gran ayuda en el estudio de estos pacientes son la TAC y la RMN, con las cuales se puede detectar lesiones mayormente en tallo encefálico y ganglios basales, aunque cabe remarcar que ninguna es específica. Infrecuentemente (como en nuestra paciente) existen reportes en los que la RMN ha mostrado lesiones expansivas que sugieren neoplasia y que se resolvieron con el tratamiento, al igual que en nuestro caso (18).

El estudio del líquido cefalorraquídeo muestra generalmente una pleocitosis moderada a predominio linfocítico y un leve incremento de la concentración de proteínas.

El tratamiento del compromiso neurológico consiste en el uso de corticoides a dosis altas y cuando existe una pobre respuesta a los esteroides se recomiendan citotóxicos. El tratamiento con clorambucil a la dosis de $0.1-0.2 \mathrm{mg} / \mathrm{kg} / \mathrm{d}$ puede controlar y previnir episodios de encefalopatía en la mayoría de paciente (19); sin embargo, su toxicidad medular y potencial oncogénico pueden limitar su uso, por lo que algunos autores recomiendan utilizar azatioprina (menor toxicidad que clorambucil) con la cual se ha reportado beneficio terapeútico aunque mayormente del compromiso ocular $(12,20)$.

Esta paciente es un caso de NeuroBehcet con imagen pseudoturmoral en la RMN cerebral, el que se resuelve con el tratamiento. Este reporte sirve para ilustrar la diversidad patológica de las lesiones del sistema nervioso central en Behcet, llegando a ser el primer caso pediátrico descrito en nuestro medio con esta complicación. 
Agradecimientos: A RESOMASA.

\section{REFERENCIAS BIBLIOGRAFICAS}

1.Weshsler B, Piette J. Behcet’s disease. Br J Med 1992; 304: 199-200.

2.Chou S. Behcet’s syndrome En: Schumacher R, Klippel J. Koopman Weds: Primer on the Rheumatic Diseases. 10 th ed. Arthritis Foundation. Atlanta GA. 1993

3.Shimizu T, Ehrich G, Inaba G, et al. Behcet's Disease Semin Arthr Rheum 1979; 8: 233-60.

4.O’Duffy J, Goldstein N. Neurological involvement in seven patients with Behcet's disease. Am J Med 1976; 61: 170-78.

5.Andrade L, Hidalgo J, Villegas A, et al. Enfermedad de Behcet con manifestaciones oculares y neurológicas. Libro de Resúmenes VI Congreso Nacional de Reumatología. Lima 1994.

6.García M, Ugarte A. Enfermedad de Behcet complicada con aneurisma de aorta torácica. Libro de Resúmenes VI Congreso Nacional de Reumatología. Lima, 1990.

7.Alonso G, Alonso E, Heinnicke M. Sindrome de Behcet: Reporte de un caso. Libro de Resúmenes VIII Congreso Peruano de Reumatología. Cuzco, 1994.

8.Acevedo E, Sedano C, Sánchez C, et al. Sindrome de Behcet en nuestro medio. Reporte de cuatro casos y revisión de la literatura. Bol Asoc Per Reumatol 1991; 10: 916.

9.Castillo S, Caderón J. Sindrome de Behcet. Reporte de tres casos en el Hospital Central de la PNP. Libro de Resúmenes VIII Congreso Peruano de Reumatología. Cuzco, 1994.

10.International Study Group for Behcet's disease: Criteria for diagnosis of Behct's disease. Lancet 1990; 335: 1078-80.

11.Chajck T, Fainaru M. Behcet's disease: report of 41 cases and a rewiew of the literature Medicine 1975; 54: 179-96.

12.Serdaroglu P, Yazici H, Ozdemir C. et al. Neurologic involvement in Behcet's syndrome. Arch Neurol 1989; 46: 265-69.

13.Kim D, Chang S, Bang D, et al. Clinical analysis of 40 cases of childhood on set Behcet's disease. Periatr Dermatol 1994; 11: 95-101. 
14.Kone-Paint I, Bernard J. Behcet's disease in children in France. Arch Fr Pediatr 1993 ; 50: 561-65.

15.Kozin F, Haughton V, Bernhard GC. Neuro-Behcet disease: Two cases and neuroradiologic findings. Neurology 1977; 27: 1148-52.

16.Bertol V, Ara JR, Oliveros A, et al. Neuro-Behcet. Estudio clínico de 9 pacientes. Neurología 1992; 7: 10-4.

17.Samoud A, Jebnoun Soud M, et al. Neurobehcet. Arch Pediatr 1994; 1: 162-5.

18.Kermode A, Plant G, Mac Manus D, et al. Behcet’s disease with slowly enlarging midline mass on MRI: resolution following steroid therapy. Neurology 1989; 39: 125152.

19.O’Duffy J, Robertson D, Goldstein N. Chlorambucil in the treatment of uveitis and meningoencephalitis of Behcet's disease. Am J Med 1984; 76: 75-84.

20.Yazici H, Pazorli H, Barnes C, et al. A controlled trial of azathioprine in Behcet's syndrome. N Engl J Med 1990; 322: 281-85.

\section{Correspondencia:}

Dra. Patricia Campos

Universidad Peruana Cayetano Heredia

Honorio Delgado 430 Lima

Apartado Postal 4314. 